Voix et Images

voixetimages

\title{
Bibliographie de Naïm Kattan
}

\section{Lucie Robert}

Volume 11, numéro 1, automne 1985

\section{Naïm Kattan}

URI : https://id.erudit.org/iderudit/200536ar

DOI : https://doi.org/10.7202/200536ar

Aller au sommaire du numéro

\section{Éditeur(s)}

Université du Québec à Montréal

\section{ISSN}

0318-9201 (imprimé)

1705-933X (numérique)

Découvrir la revue

Citer ce document

Robert, L. (1985). Bibliographie de Naïm Kattan. Voix et Images, 11(1), 45-54. https://doi.org/10.7202/200536ar d'utilisation que vous pouvez consulter en ligne.

https://apropos.erudit.org/fr/usagers/politique-dutilisation/ 


\title{
Bibliographie de Naïm Kattan
}

\author{
par Lucie Robert, Université Laval
}

Les principales divisions de cette bibliographie sont les suivantes:

\section{OEUVRES}

A. VOLUMES ET CHAPITRES DE VOLUMES

B. ARTICLES DE PÉRIODIQUES

\section{SUR NAİM KATTAN ET SUR SON GEUVRE}

A. SUR NAIIM KATTAN

B. SUR SON GEUVRE

1. Adieu Babylone; 2. Dans le désert ; 3. Le Désir et le pouvoir; 4. Écrivains des Amériques; 5 . La Fiancée promise; 6. Les Fruits arrachés; 7. Les Juifs et la communauté française; 8. La Mémoire et la promesse; 9. The Neighbour and other stories (v. aussi Dans le désert et la Traversée): 10. Le Réel et le théâtral; 11. La Reprise; 12. Le Rivage; 13. Le Sable de l'île; 14. Théâtre I; 15. La Traversée (v. aussi The Neighbour and other stories); 16. Divers.

\section{I - OEUVRES}

\section{A - VOLUMES ET CHAPITRES DE VOLUMES}

Les Juifs et la communauté française. Premier cahier du cercle juif de langue française, publié sous la direction de Naïm Kattan, Montréal, les Éditions du Jour, (1965), 136 p. (Les Idées du Jour, D-17).

V. "Introduction", p. 7-10, et "Mordecai Richler», p. 47-54. "Mordecai Richler» parut d'abord en anglais, sous le titre "Mordecai Richler, craftsman or artist», dans Canadian Literature, 21 (Summer 1964, p. 46-5I, et fut reproduit en français, dans Écrivains des Amériques, t. II, p. 45-57.

Juifs et Canadiens. Deuxième cahier du cercle juif de langue française, publié sous la direction de Naïm Kattan. Montréal, Éditions du Jour, (1967), 132 p. (V. "Préface», p. 7-8, et «les Juifs du Canada», p. 37-47.)

"Montréal a l'âge de raison?", dans l'Étoile de David et la Fleur de lys. Troisième cahier du cercle juif de langue française, publié sous la direction de V.M.H. Rodriguez, Montréal, Éditions du Jour, (1969). 195 p. (V. p. 115-121).

Parut d'abord en anglais, sous le titre "Montreal comes of age», traduction de Iron Owen. The Tamarack Review, 47 (Spring 1968), p. 76-80.

Le Réel et le théâtral. Essai, Montréal, Éditions HMH, 1970, 188 p. (Constantes, no 23); Paris, Denoël, 1971 (Les Lettres nouvelles).

Un extrait parut dans le Devoir, LX. 265 (14 novembre 1969), p. XIII. Reality and Theatre, traduction de Alan Brown, (Toronto), Anansi, (1972), viii, 142 p.

“Le Roman canadien-anglais". dans Archives des lettres canadiennes, $t$. III, le Roman canadienfrançais, Montréal, Fides, (1971), p. 257-264.

Dans André ELBAZ, Seuls, textes de Elie Wiesel et Naïm Kattan, Montréal, (l'Auteur), 1971 ( 1 étui, $26 \mathrm{f}$. volantes et 20 ill.) 
Écrivains des Amériques, Montréal, Éditions HMH, 3 vol.: t. I : les États-Unis, (1972), 243 p. (Constantes); Hurtubise HMH, 271 p. (Reconnaissances); t. II : le Canada anglais, (1976), 207 p. (Constantes); t. III : l'Amérique latine, (1980), 165 p. (Constantes).

"Saul Bellow" parut d'abord dans Revue de l'université Laval, XVII, 4 (décembre 1962), p. 316-334; "Philip Roth, écrivain américain", dans Liberté. IV, 1, no 29-30 (janvierfévrier 1964), p. 53-66; "Bernard Malamud" et "James Baldwin», dans Écrits du Canada français, XVII (1964), p. 85-135, sous le titre «Deux écrivains américains»; "Brian Moore», dans Canadian Literature, no 18 (Autumn 1963), p. 30-39.

La Discrétion. La Neige. Le Trajet. Les Protagonistes. Théâtre I, Montréal, Leméac, (1974), 136 p. (Théâtre canadien, no 30).

«Les Protagonistes» parut d'abord dans Écrits du Canada français, XXXV (1972). p. 65-98.

Dans le désert. Nouvelles, Montréal, Leméac, (1974), 153 p. (Roman québécois, no 9). "Le Tableau" parut d'abord dans Liberté, XVI, 3, no 93 (mai-juin 1974), p. 75-81 et "l'Hôtel", dans le Devoir. LVI, 231 (5 octobre 1974), p. 14-15. Quelques nouvelles sont reproduites dans The Neighbour and other stories, traduction de Judith Madley et Patricia Claxton. Toronto, McClelland \& Stewart, 1982, 183 p. (New Canadian Library, no 165). "Abu Nuwas Street" parut d'abord dans Journal of Canadian Fiction, no 17-18 (1976), p. $50-56$.

Adieu Babylone. Roman, Montréal, la Presse (1975), 238 p.; Paris, Julliard, 1976.

Un extrait parut dans le Maclean, XV. 6 (juin 1975), p. 20-21, 38-40. 42-44. Farewell Babylon. traduction de Sheila Fischman. Toronto, McClelland \& Stewart, 1976. 191 p.; New York, Tapienger, 1980, 191 p.

"L'Influence américaine sur le théâtre du Québec", dans Archives des lettres canadiennes, t. V, le Théâtre canadien-français. Montréal. Fides. (1976), p. 431-435.

La Traversée. Nouvelles. illustrations de Louise Dancoste, Montréal, HMH, (1976), $152 \mathrm{p}$. (L'Arbre).

«Le Substitut» et «les Bagages»parurent d'abord dans Écrits du Canada français, XL (1976), p. 135-150. Quelques nouvelles sont reproduites dans The Neighbour and other stories, traduction de Judith Madley et Patricia Claxton. Toronto, McClelland \& Stewart. (1982), 183 p. (New Canadian Library, no 165).

"Baggage" parut d'abord dans The Tamarack Review, 75 (Fall 1978), p. 8-14, et «Island Bookseller", dans Saturday Night, XCIV, I (January-February 1979), p. 42-45.

Les Fruits arrachés. Roman. Montréal, HMH. (1977), 230 p. (L'Arbre).

Un extrait parut dans le Devoir. LXIX. 227 (1er octobre 1977), p. 17. Paris Interlude, traduction de Sheila Fischman. Toronto. McClelland \& Stewart. (1979). 208 p.

"Réalité culturelle au Canada", dans l'Enseignement des langues ê la réalité canadiennel Language teaching and the Canadian context. Actes du se colloque de l'Association canadienne de linguistique appliquée, publié sous la direction de Jean-Guy Savard, Québec, Centre international de recherche sur le bilinguisme, 1978, 60, x p. (V. p. 1-5.)

La Mémoire et la promesse. Essai. Montréal, HMH, (1978), 160 p. (Constantes, no 37); Paris, Denoël, (1979), 160 p.

Un extrait parut sous le titre "le Lieu et l'Espace", dans Liberté, XVI, 3, no 93 (mai-juin 1974), p. 38-52.

Le Rivage. Nouvelles, Montréal, HMH, (1979), 179 p. (L'Arbre).; Paris. Gallimard, (1979), 179 p. (nrf).

Le Sable de l'île. Nouvelles, Montréal, HMH, (1981), 222 p. (L'Arbre); Paris, Gallimard, (1981), 177 p. (nrf).

"Les yeux fermés», parut d'abord dans Liberté, XXI, 3, no 123 (mai-juin 1979), p. 63-70.

La Fiancée promise. Roman. Montréal. HMH. (1983). 231 p. (L'Arbre).

Le Désir et le pouvoir, Montréal, HMH, (1983), 209 p. (Constantes, no 42).

Une partie parut d'abord dans Écrits du Canada français, XLVI (1982), p. 139-154.

La Reprise. Nouvelles, Montréal, HMH, (1985). 233 p. (L'Arbre). 


\section{B - ARTICLES DE PÉRIODIQUES 2}

«Les Juifs et la langue française», Relations, 211 (juillet 1958), p. 179-180.

"D'un monde à l'autre. Souvenirs», Écrits du Canada français, IX (1961), p. 129-147.

«Le Brésil: terre de toutes les races». Cité libre, XIII, 45 (mars 1962), p. 6-12.

"La Femme orientale», Magazine Maclean, II, 8 (août 1962), p. 14, 45-47.

«L'Alliance pour le progrès: un an après», Cité libre, XIII, 10 (octobre 1962), p. 13-14.

"Montreal Letter. (Traduction de Joyce Marshall)", The Tamarack Review, 30 (Winter 1964), p. $48-52$.

"Lettre de Montréal", Canadian Literature. 22 (Autumn 1964), p. 50-54.

«L'Arrivée. Récit», Écrits du Canada français, XIX (1965), p. 219-246.

«Letter from Montreal. The new elite and the institutions", The Tamarack Review, 34 (Winter 1965), p. 57-64.

"Écrivains et religion établie», Maintenant, 37 (janvier 1965), p. 27.

"La Jeunesse américaine: la génération tendue», Cité libre, XV, 3 (mars 1965), p. 21-23.

«Lettre de Montréal. Une nouvelle littérature?», Canadian Literature, 24 (Spring 1965), p. 47-51.

"Le Crime et la société américaine», Cité libre, XVII, 5 (mai-juin 1965), p. 24-27, 33.

"Les Groupes religieux et le centenaire du Canada». Culture, XXVI, 3 (septembre 1965). p. $323-325$.

«Lettre de Montréal. Compte rendu», Canadian Literature, 26 (Autumn 1965), p. 55-58.

"Montreal Letter. French-Canadian Plays. (Traduction de Joyce Marshall)", The Tamarack Review, 37 (Autumn 1965), p. 60-64.

"Romanciers canadiens-anglais et canadiens-français». Liberté. VIl, 6 no 42 (novembredécembre 1965), p. 479-483.

"Montreal and French-Canadian Culture. What they mean to English-Canadian Novelists", The Tamarack Review, 40 (Summer 1966), p. 40-53 (article écrit en collaboration.)

"Lettre de Montréal: Montréal, ville internationale", Canadian Literature, 30 (Summer 1966), p. 49-52. Reproduit au no 37 (Summer 1968), p. 68-71.

"Le Congrès du PEN Club à New York. Entretien avec Arthur Miller", le Devoir. LVII, 146 (23 juin 1966), p. 23.

«Le Happening, une nouvelle forme de théâtre?», le Devoir, LVIl, 156 (7 juillet 1966), p. 8.

"Le Nationalisme change de visage», Cahiers de Cité libre, XVII, 1 (septembre-octobre 1966). p. 86-90.

"Ả Edmonton. Où va la littérature canadienne?», le Devoir, LVIII, 79 (5 avril 1967), p. 8. "L'Édition au Canada français", Canadian Literature, 33 (Summer 1967), p. 16-24.

"Marshall McLuhan», Liberté, IX, 5, no 53 (septembre-octobre 1967), p. 15-20.

"Une question, des réponses (Qu'appelleriez-vous érotisme?)», Liberté. IX, 6, no 54 (novembre-décembre 1967), p. 30-38 (V. p. 31-32.)

"André Laurendeau. Nouveaux témoignages», le Devoir, LIX, 131 (4 juin 1968), p. 4.

"L'Immigrant de langue française et son intégration à la vie canadienne", Écrits du Canada français, XXV (1969), p. 173-247.

"Le Théâtre et les dramaturges à Montréal», Canadian Literature, 40 (Spring 1969), p. 43-48. "L'Écrivain canadien et les échanges internationaux", Liberté, XII, 3, no 69 (mai-juin 1970), p. $117-121$.

"Éducation artistique et prise de conscience du droit à la culture», Culture, XXXI, 3 (septembre 1970), p. 193-204.

"La Belle Bête, de Marie-Claire Blais», Culture, XXXII, 1 (mars 1971), p. 400-404.

"L'Influence américaine sur le roman canadien», Société rovale du Canada. Mémoires, 4 " série, t. II, section I, XI (1973), p. 81-84.

"Le Roman canadien-français». Bulletin de la Société des écrivains canadiens, IV, 2 (mars 1973), p. 5. 
"La Francophonie canadienne: une civilisation réinventée?", Revue de l'Association canadienne d'éducation de langue française, II, 2 (mai 1973), p. 17-19.

"Littérature de (sic) Québec: Langue et identité»,Canadian Literature, 58 (Autumn 1973), p. 61-63.

"Ả Londres, peut-on parler anglais?», le Devoir, LXIV, 276 (29 novembre 1973), p. 4.

"Littérature et Idéologie». Études littéraires. VI, 3 (décembre 1973), p. 339-344.

“Explorations. Brasilia vaincue par les Brésiliens", le Maclean, X1II, 12 (décembre 1973), p. 12-14.

“Explorations. En Bulgarie même les tomates sont rouges», Le Maclean, XIV, 4 (avril 1974), p. 9-10, 12 .

"L'Algérie des survivants», le Maclean, XIV, 9 (septembre 1974), p. 48-50, 52.

"La Rose est une fleur", le Maclean. XIV, 10 (octobre 1974), p. 26-28, 34, 39-40.

"La Culture et l'État. Discours de réception à la Société royale du Canada", Société royale du Canada. Présentation, XXX (1974-1975), p. 125-134.

Reproduit dans le Devoir, LXVII, 113 (17 mai 1975), p. 33.

"La Parole aux traducteurs (...) Un travail sur sa propre langue", le Devoir, LXVII, 44 (22 février 1975), p. 15-16.

"L'Exil recommence», Études littéraires, VIII, 1 (avril 1975), p. 21-31.

"L'Espace dans la littérature québécoise", Société royale du Canada. Mémoires, $4^{\mathrm{e}}$ série, XIV (1976), p. 127-129.

"Le Dîner. Conte", Liberté, XVIII, 1, no 104 (mars-avril 1976), p. 56-62.

“La Dimension culturelle des deux solitudes», Études internationales, VIII, 2 (juin 1971), p. $337-341$.

"Sixième séance. (Rencontre québécoise internationale des écrivains)», Liberté. XIX, 4, no 112 (juillet-août 1977), p. 270-311 (passim).

“Les Deux Berlin», Actualité, III. 6 (juin 1978), p. 68, 70, 72.

"Écrivain et Lecteur. (Rencontre québécoise internationale des écrivains. Débats)", Liberté, XX, 4-5, no 118-119 (juillet-octobre 1978), p. 42-175 (passim.)

"Ontologie, esthétique et cuvre d'art littėraire», Philosophiques, V, 2 (octobre 1978), p. 261-269.

"Littérature et Réalité. (Rencontre québécoise internationale des écrivains. Débats)», Liberté. XXI, 4-5, no 124-125 (juillet-octobre 1979), p. 67-163 (passim).

«Littérature mondiale. Littérature des nations», Société royale du Canada. Mémoires, $4^{\mathrm{e}}$ série, XVIII (1980). p. 161-164.

"Le Sacré, la Littérature et le Profane. (Rencontre québécoise internationale des écrivains. Débats)", Liberté, XXI1I, 4, no 136 (juillet-août 1981), p. 5-119 (passim.)

"Garnisons du Canada: entre la mémoire et la promesse", Voix el Images. VI. 2 (hiver 1981), p. $235-244$.

"Table ronde. Image rétrospective du rôle d'une académie des lettres», Société royale du Canada. Mémoires, $4^{\mathrm{e}}$ série, XX (1982), p. 182-194. (V. p. 191-192.)

«Le Pari faustien», Liberté, XXIV. 5, no 143 (octobre 1982). p. 107-123.

"Peut-on traduire les civilisations?", Meta. Journal des traducteurs, XXVII, 4 (décembre 1982), p. $401-403$.

«Le Jardin rêvé», Canadian Literature, 100 (Spring 1984), p. 164-170. 


\section{SUR NAIM KATTAN ET SUR SON GEVRE}

A. SUR NAİM KATTAN

(ANONYME), “Naïm Kattan», la Presse, XCII, 47 (25 février 1976), p. E-9.

- . "Our only Arab-Jewish-French-Canadian author», Saturday Night, XCIV, 1 (JanuaryFebruary 1979), p. 9.

- "Naïm Kattan: "Canada deals with its contradictions by expressing them loudly'", Quill and Quire, XLVII, 5 (May 1981), p. 9-11.

CANTIN, Pierre, Normand HARRINGTON et Jean-Paul HUDON, Bibliographie de la critique de la littérature québécoise, Ottawa, Centre de recherche en civilisation canadiennefrançaise. 1979, 5 vol. x, 1254 p. (Pagination continue. Documents de travail).

DEY, Olga, "Naïm Kattan: writer», Canadian Author and Bookman, LV, 4 (et) LVI, 1 (Summer-Fall 1980), p. 24-25.

FULFORD, Robert, "The Canada Council at twenty-five", Saturday Night, XCVII, 3 (March 1982), p. $34-45$.

GREENSTEIN, Michael. «Naïm Kattan», dans William TOYE (editor). The Oxford Companion to Canadian Literature, Toronto, Oxford and New York, Oxford University Press. 1983,843 p. (V. p. $404-405$ )

HAMEL. Réginald, John HARE et Paul WYCZYNSKI, Dictionnaire pratique des auteurs québécois. Montréal, Fides, (1976), 723 p. (V. p. 369-370.)

LEDUC, Jean, Naïm Kattan. Essai de poésie rough and tough aléatoire flanqué de Parti pour le (rou, Montréal, Éditions Cul Q, (1977), (n. p.) (Mium, Mium, no 15).

PILON. Jean-Guy, “Présentation de Naïm Kattan», Société royale du Canada. Présentation. XXX (1974-1975), p. 119-124.

SIMPSOM, Leo, “Conversation with Naïm Kattan», Quill and Quire, XLII, 17 (December 1976). p. 9-10, 36.

UNION DES ECRIVAINS QUÉBÉCOIS, Dictionnaire des écrivains québécois contemporains, recherche et rédaction Yves Légaré, Montréal, Québec/Amérique, (1983), 399 p. (V. p. 211-212.)

\section{B - SUR SON CEUVRE}

\section{Adieu Babylone}

(ANONYME), “Ouvrages reçus", le Canada français (Saint-Jean), CXVI, 34 (21 janvier 1976). p. 47.

- "Adieu Babylone", le Livre canadien, VII, 272 (septembre 1976).

APPENZELL. Anthony, "The Modes of maturity», Canadian Literature, 72 (Spring 1977) p. $69-74$ (V. p. 72. )

BASILE, Jean, «Roman/Adieu Babvlone de Naïm Kattan. Tout Juif porte en lui un arabe qui sommeille", le Devoir, LXVIII, 7 (10 janvier 1976), p. 16.

BUSCH, B.J., "Farewell, Babylon", The Canadian Reader, XVII. 12 (December 1976), p. 12.

CHASSÉ, Denis, "Naïm Kattan. Dans le désert et Adieu Babylone», Livres et Auteurs québécois, (1975), p. 53-56.

FREEDMAN, Adele, “Farewell, Babylon», Macleans, LXXXIX, 16 (September 20, 1976), p. $65-66$.

GODBOUT, Jacques, "Livres. Ces voyages qui forment la jeunesse", le Maclean, XVI, 4 (avril 1976), p. 12.

HORNBECK, Paul, «Farewell, Babylon», Quill and Quire, XLII, 14 (October 18, 1976), p. 7-8.

MANTZ, Douglas, "Farewell, Babylon ", Canadian Fiction Magazine, 24-25 (Spring-Summer 1977), p. 179-181.

MARTEL, Réginald, "Autour et à propos d’un livre», la Presse, XCII, 2 ( 3 janvier 1976), p. $\mathrm{C}-4$.

OWEN, I.M., "Bridge of Tongues. Why an Arabic-speaking, Baghdad-born Jew is a perfect guide to the modern Canadian experience», Books in Canada, V, 12 (décembre 1976). p. 5-6. 
PAGE, Raymond, “Literary Year in Québec. 1975-76", Chelsea Journal, III, 2 (mars-avril 1977), p. 93-96. (V. p. 96.)

PIAZZA, François, "Adieu Babylone», Montréal-matin, XLVI, 179 (28 décembre 1975), p. 9.

POULIN, Gabrielle, "Letters in Canada (...) Roman, récits, nouvelles, contes", University of Toronto Quarterly, XLV, 4 (Summer 1976), p. 326-333. (V. p. 331.)

ROYER, Jean, "Apprendre à s'enrichir de la culture des autres», le Soleil, LXXX, 182 (31 juillet 1976), P. D-1 - D-2.

SPETTIGUE, D.O., "Farewell, Babylon", Queen's Quarterly, LXXXIV, 3 (Autumn 1977), p. 510-511.

SUTHERLAND, Ronald, "Mind Broadening", The Canadian Forum, LVI, 669 (March 1977), p. 36.

THÉRIAULT, Jacques, “Un roman de Naïm Kattan lève le voile sur Bagdad», le Devoir, LXVIII, 4 (7 janvier 1976), p. 8.

TOURNIER, Michel, préface à l'édition Julliard; reproduit dans la Presse, XCII, 150 (12 juin 1976), p. E-2.

VANASSE. André, «les Nouvelles Voix romanesques. Naïm Kattan: la Traversée... de Babylonè, Lettres québécoises, 5 (février 1977), p. 8-10.

VAN ROEY-ROUX, Françoise, la Littérature intime du Québec, Montréal, Boréal Express, (1983). 254 p. (V. p. 188-222.)

2. Dans le désert. (Voir aussi The Neighbour and other stories).

(ANONYME), "Dans le désert», le Livre canadien. VI, 138 (avril 1975).

BASILE, Jean. "la Fiction. Dans le désert, recueil de nouvelles universaliste», le Devoir, LXVII, 44 (22 février 1975), p. 22.

BELLEMARE. Pierre D., "Dans le désert, de Naïm Kattan. Entre le perdu et le possible", le Bien public, LXII, 52'( 1 et 2), (3 et 10 janvier 1975), p. 7.

CHASSÉ. Denis, "Naïm Kattan. Dans le désert et Adieu Babylone», Livres et Auteurs québécois, (1975). p. 53-56.

GODBOUT, Jacques, "Livres. Ces voyages qui forment la jeunesse», le Maclean, XVI, 4 (avril 1976), p. 12.

HÉBERT, François, «le Noir et le Blanc, le Bleu et le Rouge», Études françaises, XI, 2 (mai 1975), p. 111-119. (V. p. 118.)

MARTEL, Réginald, “l'Instant qui nie le temps", la Presse. SCI. 15 (18 janvier 1975), p. E-3.

PIAZZA, François, "Pour mettre sous l'arbre», Montréal-matin, XLV, 170 (22 décembre 1974), p. 23.

RENAUD, André, «Parlez-moi... de livres. Dans le désert», le Droit, LXII, 272 (15 février 1975), p. 16.

TREMBLAY, Robert, "Lettres. Dans le désert de Naïm Kattan», le Soleil, LXXIX, 25 (25 janvier 1975), p. D-8.

\section{Le Désir et le pouvoir}

LAR UE. Monique, "Une chevauchée planétaire», Spirale, 39 (décembre 1983), p. 12.

LE BLANC, Alonzo, "Essais. Le Désir et le pouvoir», Québec français, 51 (octobre 1983), p. 15-16.

VIGNEAULT, Robert, "l'Essai, cette passion du sens", Lettres québécoises, 31 (automne 1983), p. $65-68$.

\section{4. Écrivains des Amériques}

BASILE, Jean, "La Littérature de l'autre solitude», le Devoir, LXVIII, 107 (8 mai 1976), p. 15 (t. I et II.)

BOURQUE, Paul-André, "Écrivains des Amériques, de Naìm Kattan», Livres et Auteurs québécois, 1972, p. 209 (t. I.)

GODBOUT, Jacques, "les Livres. Les Fesses de la fille avant tout", le Maclean, XIII, 3 (mars 1973), p. 62 (t. I.) 
HODGSON, Richard G., "Analyst. Naïm Kattan, Le Rivage and Écrivains des Amériques, t. III ", Canadian Literature, 90 (Autumn 1981). p. 151-153.

JACQUES, André, "Naïm Kattan. Écrivains des Amériques, t. III: I'Amérique latine», Livres et Auteurs québécois, (1980), p. 197-199.

KATTAN, Naïm. “Découvrir et Redécouvrir», le Devoir, LXII, 249 (28 octobre 1972), p. XLV.

MÉLANÇON, Robert, "Naïm Kattan. Écrivains des Amériques II", Livres et Auteurs québécois, (1976), p. 233-234.

MOREAU. Jean-Marie. "Écrivains des Amériques tome I: les États-Unis; tome II : le Canada anglais", Nos livres, VIII, 218 (juin-juillet 1977).

OUELLETTE-MICHALSKA, Madeleine, "Naïm Kattan. Voix des littératures d'Amérique», le Devoir, LXXI. 157 (12 juillet 1980), p. 15 (t. III.)

ROCHER, Guy, "l'Angoisse de l'homme étatsunien vue par Naïm Kattan», le Devoir, LXIV, 236 (13 octobre 1973). p. 15 (t. I.)

TREMBLAY, Robert, "les Parutions récentes", le Soleil, LXXVII, 3 (30 décembre 1972), p. 48.

\section{La Fiancée promise}

MARTEL, Réginald, "Naïm Kattan: les femmes du pays froid", la Presse, C, 53 (3 mars 1984), p. E-3.

PELLERIN, Gilles, "les Fiancées de la Terre promise. La Fiancée promise», Lettres québécoises, 33 (printemps 1984), p. 92.

6. Les Fruits arrachés

(ANONYME), "les Fruits arrachés», le Québec en bref, XII, 2 (février 1978), p. 18.

- , "The Editors recommend", Books in Canada, IX, I (January 1980), p. 25.

ARĖS, Raymond, “Naïm Kattan. Les Fruits arrachés», Livres et Auteurs québécois, (1976). p. $78-79$.

BENAZON, Michael, "An Iraqi in Paris. Paris Interlude", The Canadian Forum, LIX, 695 (December 1979-January 1980), p. 40-41.

CZARNECKI. Mark, "Stale Baguettes and Love Affairs in Paris", Maclean's, XCII, 43 (October 1979), p. 58.

DEY, Olga, «Naïm Kattan: Writer», Canadian Author and Bookman, LV, 4 (et) LVI, 1 (Summer-Fall 1980), p. 24-25.

DORION, Gilles, "les Fruits arrachés", Québec français, 29 (mars 1978), p. 7.

DORSINVILLE, Max, «Idéologies: André-J. Bélanger, Ruptures et Constantes; Naïm Kattan, les Fruits arrachés; Pierre Séguin, Caliban", Canadian Literature. 84 (Spring 1980). p. 84-90.

DROLET, Gilbert, "les Fruits arrachès», Quill and Quire, XLIV, 10 (July 1978), p. 13.

FRENCH, William, "Novelist charts rites of passage in sexual sea". The Globe and Mail, CXXXVI, 40, 488 (September 13, 1979), p. 13.

GAUVIN, Lise, «Letters in Canada (...) Romans, récits», University of Toronto Quarterly. . XLVII, 4 (Summer 1978), p. 338-346. (V. p. 341.)

GROSSKURTH, Phyllis, "The Young who wanted to love», Saturday Night, XCV, 1 (JanuaryFebruary 1980), p. 50.

MÉLANÇON, Robert, "Entre autres, deux romans», le Devoir. LXIX, 17 (21 janvier 1978), p. 33.

MOREAU, Jean-Marie, «les Fruits arrachés», Nos livres, IX, 366 (octobre 1978).

O'CONNOR. John. "Letters in Canada (...) Translations", University of Toronto Quarterly. XLIX, 4 (Summer 1980), p. 383-399. (V. p. 386.)

OUELLETTE-MICHALSKA. Madeleine, "Livres. À chacun son nouveau monde», Châtelaine, XIX, 4 (avril 1978), p. 8

SCOBIE, Stephen «Far from the eyes of night: Paris Interlude». Books in Canada. VIII, 10 (December 1979), p. 8.

Voir aussi The Toronto Star, September 15, 1979, p. G-7; The Calgary Herald, October 20. 1979. p. B-20; Winnipeg Free Press. November 10. 1979. Insert, p. 6 (non vidi) 


\section{VOIX \& IMAGES, VOL. XI, NO 1 (AUTOMNE 85)}

\section{Les Juifs et la communauté française}

BOSCO. Monique, "Un grand cahier du Cercle juif", le Magazine Maclean, V, 12 (décembre 1965), p. 67.

BOURNEUF, Roland, "Divers. Les Juifs et la communauté française". Livres et Auteurs canadiens, (1967), p. 154.

\section{La Mémoire et la promesse}

(ANONYME), "la Mémoire et la promesse», le Québec en bref, XII, 6 (juin 1978), p. 20.

PARÉ. François, «Entre les lignes. Naïm Kattan: 'L'Amérique n'a pas tenu promesse' », le Droit, LXVI, 168 (14 octobre 1978), p. 21.

ROYER, Jean, «Naïm Kattan», LXIX, 156 (8 juillet 1979

WEINMANN, Heinz, "Naïm Kattan. La Mémoire et la promesse», le Devoir, LXIX, 156 (8 juillet 1978), p. 17.

9. The Neighbour and other stories (v. aussi Dans le désert et la Traversée).

CURRIE, Rod, "Neighbour' a gem of a collection". The Montreal Gazette, CCV (June 19, 1982), p. B-8.

FRENCH, William, «Kattan captures futile search for one's heritage», The Globe and Mail, CXXXVIII, 41, 234 (February 4, 1982), p. E-1.

GAVER, Stephen, "The Neighbour and other stories", Quill and Quire, XLVIII, 4 (Sprng 1982), p. 26-28.

GRADY, Wayne, "The other Canadian. Though cosmopolitan in content Naïm Kattan's stories explore a familiar Canadian duality.: the plight of the stranger in its own land", Books in Canada. XI. 5 (May 1982), p. 9-11.

MEZEI, Kathy, "Letters in Canada (...) Translations", University of Toronto Quarterly. LII, 4 (Summer 1983), p. 385-397. (V. p. 392-393.)

Voir aussi The Vancouver Sun, March 12, 1982, p. 36; The Toronto Star, May 1st, 1982, p. H-10 (non vidi).

\section{Le Réel et le théâtral}

(ANONYME), «le Prix France-Canada», Vient de paraitre, VIIl, I (mars 1972), p. 18.

BASILE, Jean, "le Réel et le théâtral, de Naïm Kattan», le Devoir, LXI, 55 (7 mars 1970), p. 12.

BEAULIEU, Ivanhoé, “'l'Acteur, c'est l'occidental poussé à l'extrême' (Naïm Kattan)", le Soleil, LXXIII, 58 (7 mars 1970), p. 46.

CONQUER, Thérèse, "Entrevue avec Naïm Kattan", le Droit, LIX, 228 (24 décembre 1971),

p. 15.

ÉTHIER-BLAIS, Jean, "Domaine québécois/le Réel et le théâtral, de Naïm Kattan. De nombreuses questions, toutes intéressantes", le Devoir, LXI, 72 (28 mars 1970), p. 13.

GROSJEAN. Jean, préface, p. 9-10.

HOULE. Benoît, "le Prix France-Canada pour le Réel et le théatral", le Soleil, LXXIV, 261 (6 novembre 1971), p. 65.

MORICE, Louis, “le Réel et le théâtral, de Naïm Kattan", Livres et Auteurs québécois. (1970), p. 152-153.

RACINE, Claude, «Naïm Kattan. Le Réel et le théâtral», Culture, XXXI, I (septembre 1970), p. 264-265.

RICHER, Julia, «l'Édition québécoise en quelques lignes (...) Le Réel et le théátral I'Action nationale, LIX. 10 (juin 1970), p. 996-997.

TARRAB, Gilbert, “Du silence claudélien à l'Orient de Kattan", la Presse, LXXXVI, 84 (I1 avril 1970), p. 29.

VIGNEAULT, Robert, "Chroniques. L'essai», Études françaises, VII, 1 (février 1971), p. 87-102. (V. p. 92-96.)

WOODCOCK. George. "Reviews. Reality and Theater". Canadian Literature, 53 (Summer 1972), p. 3-7. 


\section{La Reprise}

BEAULIEU, Ivanhoé, "Un clin d'œil au désespoir. Lettres québécoises", le Devoir, LXXXVI, 144 (22 juin 1985), p. 35.

\section{Le Rivage}

(ANONYME), «le Rivage», Points. III. 2 (été 1979), p. 6.

BEAULIEU, Benoît, "Naïm Kattan. Le Rivage», Livres et Auteurs québécois, (1979), p. 54-55.

HODGSON, Richard G., "Analyst. Naïm Kattan, le Rivage et Écrivains des Amériques, t. III". Canadian Literature. 90 (Autumn 1981), p. 151-152.

MÉLANÇON. Robert, "les Bonnes Nouvelles de Naïm Kattan», le Devoir, LXX, 186 (11 août 1979), p. 14.

MOREAU, Jean-Marie, “le Rivage», Nos livres, X, 263 (août-septembre 1979).

OUELLETTE-MICHALSKA, Madeleine, "Livres. Rester jeunes toute la vic?m, Châtelaine. XX. 11 (novembre 1979), p. 10.

\section{Le Sable de l'âle}

BOIVIN, Aurélien, "le Sable de l'île", Livres et Auteurs québécois, (1981), p. 54-55.

- "Naïm Kattan. Le Sable de l'île», Québec français, 43 (octobre 1981), p. 12-13.

BURGUET, Frantz-André, "le Sable de l'ile», le Magazine lintéraire, 170 (mars 1981), p. 69. PIAZZA, François, "le Temps personnalisè". le Devoir. LXXII, 234 ( 12 décembre 1981), p. 36.

SOUCY, Jean-Yves, «Naïm Kattan. La grande aventure de la quotidienneté», Reflets. III. 2 (janvier 1981), p. 24.

\section{Théâtre I}

(ANONYME), "la Discrétion. La Neige. Le Trajet. Les Protagonistes", le Livre canadien, V, 285 (octobre 1974).

- . "Deux pièces de Kattan à 'Premières'", le Devoir, LXIV, 126 (31 mai 1973), p. 12. (Le Trajet et l'Indiscrétion (sic).)

GAY. Paul. «Parlez-moi... de livres. La Discrétion», le Droil, XLII, 56 (ler juin 1974), p. 17.

GOBIN, Pierre, «Diction. Vision. Figuration”, Livres et Auteurs québécois, (1974), p. 165-168.

HOULE, René, «'Premières'. Le Trajet, Naïm Kattan». Ici Radio-Canada/radio. 55 (26 mai [973), p. 7.

MAILHOT, Laurent, préface, p. 7-15.

- . "Letters in Canada (...) Théâtre», University of Toronto Quarterly, XLIV, 4 (Summer 1975). p. 343-359. (V. p. 358.)

PAGE. Pierre, avec la collaboration de Renée LEGRIS et de Louise BLOUIN, Répertoire des aeuvres de la littérature radiophonique québécoise, 1930-1970. Montréal, Fides, (1977), 826 p. (V. p. 343.) (Archives québécoises de la radio et de la télévision, no 1).

RINFRET. Édouard-G., le Théâtre canadien d'expression française. Répertoire analytique des origines à nos jours. Montréal, Leméac, t. II, (1976), 404 p. (V. p. 243.)

THÉRIAULT, Jacques, «Naïm Kattan, dramaturge. Le théâtre n'est pas qu'un jeu de masque», le Devoir. LXV, 60 (14 mars 1974), p. 13.

15. La Traversée (Voir aussi The Neighbour and other stories).

(ANONYME), «la Traversée», le Québec en bref. XI, 2 (février 1977), p. 20.

AMPRIMOZ. Alexandre. "Reviews. Quebec writers: The Anatomy of solitude», The Tamarack Review, 72 (Fall 1977), p. $79-87$ (V. p. 79-82.)

BÉLANGER-POPVASSILEVA, Bagriana, "Entre les lignes. La Traversée, de Naïm Kattan. Les Chemins qui se croisent", le Droit, LXV, 70 (18 juin [977), p. 21.

BOIVIN, Aurélien, "la Traversée», Québec français, 26 (mai 1977), p. 8.

MARTEL, Réginald. «Des nouvelles, un roman et des riens», la Presse, XClI, 290 (4 décembre 1976), p. E-3.

MOREAU, Jean-Marie, "la Traversée», Nos livres, VIII, 93 (mars 1977). 
PAGE, Raymond, "Quebec Literature. An annual survey", Chelsea Journal, IV, 4 (JulyAugust 1978), p. 195-198. (V. p. 196.)

PIETTE, Alain, «Naïm Kattan. La Traversée», Livres et Auteurs québécois, (1976), p. 83.

RICARD, François, "D'Andrée Maillet à Naïm Kattan", le Devoir, LXVIIl, 295 ( 18 décembre 1976), p. 16.

ROUX, Paul, «Naïm Kattan, un excellent conteur», le Soleil, LXXX, 294 (11 décembre 1976), p. E-11.

THÉRIO, Adrien. "Des choses à dire. Deux livres à lire et deux affaires à dénoncer», Lettres québécoises, 7 (août-septembre 1977), p. 55.

VANASSE, André, "les Nouvelles Voix romanesques. Naïm Kattan: la traversée... de Babylone", Lettres québécoises, 5 (février 1977), p. 8-10.

\section{Divers}

(ANONYME), “'le Testament”», lci Radio-Canada/radio. 197 (14 février 1976), p. 6-7. (Sur une pièce inédite.)

ANCTIL, Michel, "Romanciers sud-américains", le Devoir, LXV, 9 (12 janvier 1974), p. 18. Réponse à «Romanciers de l'histoire. Les Sud-américains: trois civilisations», le Devoir. LXIV. 260 (10 novembre 1973), p. XXXI-XXXII.

CLARK. Roger (et) Rodrigue BÉRUBE, «Ả propos de Jonathan le Goéland. Une faillite intellectuelle ou un cadre traditionnel?", le Devoir, LXIV, 179 (4 août 1973), p. 15. Réponse à «les Lettres américaines. Un livre jalon: Jonathan Livingstone», le Devoir. LXIV, 156 ( 7 juillet 1973), p. 15.

ÉTHIER-BLAIS, Jean, "Essai. Naïm Kattan nous tend un miroir", le Devoir, LX, 100 ( 10 mai 1969), p. 16. (Sur "l'Immigrant de langue française au Canada (...)m.)

HOULE. René. “'la Sortie'. Ici Radio-Canada/radio, 54 (19 mai 1973). p. 8. (Sur une pièce inédite.)

— . "Premières. 'Le vol', de Naïm Kattan», Ici Radio-Canada/radio, 104 (4 mai 1974), p. 23. (Sur une pièce inédite.)

- "'la Poursuite'», Ici Radio-Canada/radio, 196 (7 février 1976), p. 22. (Sur une pièce inédite.)

1 - Il s'agit ici essentiellement de la bibliographie québécoise et de la bibliographie canadienne de Naïm Kattan. Je remercie Aurélien Boivin pour son aimable collaboration.

2 - Naïm Kattan a été rédacteur en chef du Bulletin du Cercle juif de 1960 à 1967 et y a écrit de nombreux textes rarement signés. Il a aussi écrit régulièrement dans le Devoir, de 1968 à 1984 . On trouvera la liste intégrale de ces articles dans Index du Devoir puis Index de l'actualité, aux années concernées. Il a, en outre, écrit une chronique dans Liberté, sur «les Écrits canadiens-anglais» et les "Lettres américaines". Plusieurs de ces articles de critique, non recensés ici, furent reproduits dans Écrivains des Amériques.

Lucie Robert. 